\title{
Dose-response study of the effect of a nutraceutical combining oat beta-glucan and green coffee hydroxycinnamates in overweight/ obese subjects
}

\author{
Raquel Mateos $^{1}$, Joaquin Garcia-Cordero $^{1}$, Susana Gonzalez-Ramila ${ }^{2}$, Beatriz Sarria ${ }^{1}$ and \\ Laura Bravo ${ }^{1}$ \\ ${ }^{1}$ Institute of Food Science Technology and Nutrition (ICTAN-CSIC), Madrid, Spain and \\ ${ }^{2}$ Institute of Food Science Technology and Nutrition (ICTAN-CSIC), Madrid, South Africa
}

\section{Abstract}

Obesity and its associated comorbidities (type 2 diabetes mellitus, cardiovascular disease, etc.) are a major public health concern worldwide, with prevalence of obesity affecting in Europe up to $17 \%$ of the adult population (OCED/EU 2016). Strategies to lose weight mostly relay in lifestyle changes such as restriction in food intake and increased exercise. However, these are not always easily accepted by overweight/obese subjects and their long-term efficiency is limited. Nutraceuticals and functional foods might be an alternative means to help lose weight and reduce associated cardiometabolic risk factors in affected subjects. There are different nutraceuticals/functional foods containing dietary fibre (DF) or polyphenol-rich plant extracts, but to our knowledge there is no nutraceutical combining both bioactive compounds.

The objective of the present study was to assess the efficiency on different risk makers of a dietary supplement combining a soluble DF (oat beta-glucan -BG-) and a green coffee bean extract (GCBE) rich in polyphenols (PP) in overweight/obese subject.

A randomized, parallel, blind, dose-response study was performed in 60 overweight/obese subjects divided into four groups $(\mathrm{n}=15)$ who consumed during 6 weeks two servings per day of one of four nutraceutical formulations containing low ( $3 \mathrm{~g} / \mathrm{d})$ or high $(5 \mathrm{~g} / \mathrm{d})$ doses of BG of different purity (35\& 70\%) and a fixed amount of GCBE providing $600 \mathrm{mg} / \mathrm{d}$ PP. Food intake, anthropometry and different cardiometabolic markers were assessed at the beginning and end of the intervention.

Results showed reduced levels of total cholesterol $(p=0.0001)$, LDL-cholesterol $(p=0.01)$, VLDL-cholesterol $(p=0.014)$ and triglycerides $(p=0.023)$. There were differences among both types of BG, with the $35 \%$ BG being more effective reducing lipid plasma levels than the $70 \%$ BG. Percentage of body fat significantly decreased after the intervention, the $70 \%$ BG being more efficient than the $35 \%$ BG. However, there was no effect on body weight and body mass index, which were not significantly modified

It can be concluded that DF and PP might have a synergistic effect reducing cardiometabolic risk factors in overweight/obese subjects.

Funded by Spanish State Research Agency, project AGL2015-69986-R

\section{Conflict of Interest}

There is no conflict of interest 